\title{
THE
}

$1-1-1984$

\section{Dynamic Correlation Functions for One-Dimensional Quantum Spin Systems: New Results Based on a Rigorous Approach}

Gerhard Müller

University of Rhode Island, gmuller@uri.edu

Robert E. Shrock

Follow this and additional works at: https://digitalcommons.uri.edu/phys_facpubs

Terms of Use

All rights reserved under copyright.

\section{Citation/Publisher Attribution}

G. Müller and R.E. Shrock. Dynamic correlation functions for one-dimensional quantum spin systems: new results based on a rigorous approach. Phys. Rev. B 29 (1984), 288-301.

Available at http://dx.doi.org/10.1103/PhysRevB.29.288.

This Article is brought to you for free and open access by the Physics at DigitalCommons@URI. It has been accepted for inclusion in Physics Faculty Publications by an authorized administrator of DigitalCommons@URI. For more information, please contact digitalcommons-group@uri.edu. 


\title{
Dynamic correlation functions for one-dimensional quantum-spin systems: New results based on a rigorous approach
}

\author{
Gerhard Müller and Robert E. Shrock \\ Institute for Theoretical Physics, State University of New York at Stony Brook, Stony Brook, New York, 11794
}

(Received 28 July 1983)

\begin{abstract}
We present new results on the time-dependent correlation functions $\Xi_{n}(t)=4\left\langle S_{0}^{\xi}(t) S_{n}^{\xi}\right\rangle, \xi=x, y$, at zero temperature of the one-dimensional $S=\frac{1}{2}$ isotropic $X Y$ model $(h=\gamma=0)$ and of the transverse Ising (TI) model at the critical magnetic field $(h=\gamma=1)$. Both models are characterized by special cases of the Hamiltonian $H=-J \sum_{l}\left[(1+\gamma) S_{l}^{x} S_{l+1}^{x}+(1-\gamma) S_{l}^{y} S_{++1}^{y}+h S_{l}^{z}\right]$. We have derived exact results on the long-time asymptotic expansions of the autocorrelation functions $\Xi_{0}(t)$ and on the singularities of their frequency-dependent Fourier transforms $\Phi_{0}^{\xi \xi}(\omega)$. We have also determined the latter functions by high-precision numerical calculations. The functions $\Phi_{0}^{\xi \xi}(\omega)$, $\xi=x, y$, have singularities at the infinite sequence of frequencies $\omega=m \omega_{0}, m=0,1,2,3, \ldots$, where $\omega_{0}=J$ for the $X Y$ model and $\omega_{0}=2 J$ for the TI model. In the TI case, the leading singularities in $\phi_{0}^{x x}(\omega)$ are alternately one-sided and two-sided power-law singularities, the first two of which (at $\omega=0,2 J)$ are divergent. The dominant singularities in the $X Y$ case are alternate one-sided power laws and two-sided power laws with logarithmic corrections, the first two of which (at $\omega=0, J$ ) are divergent. The singularities at higher frequencies in both models are finite and become increasingly weaker. We point out that the nonanalyticities at $\omega \neq 0$ are intrinsic features of the discrete quantum chain and have therefore not been found in the context of a continuum analysis (Luttinger model). At least the most prominent features of our new results should be observable in lowtemperature dynamical experiments on quasi-one-dimensional compounds such as the $X Y$-like substances $\mathrm{Cs}_{2} \mathrm{CoCl}_{4}$ and $\mathrm{PrCl}_{3}$ and the $S=\frac{1}{2}$ Ising-like substance $\mathrm{CsCoCl}_{3} \cdot 2 \mathrm{H}_{2} \mathrm{O}$.
\end{abstract}

\section{INTRODUCTION}

Exact results are available for the thermodynamic properties of many one-dimensional (1D) classical- and quantum-spin systems. For most of these "exactly solvable" model systems, however, no rigorous results are known for dynamic correlation functions. This is the case, for example, for the classical Heisenberg model, whose partition function has been calculated in closed form, ${ }^{1}$ and for the $S=\frac{1}{2}$ Heisenberg-Ising model, whose free energy is amenable to exact Bethe-Ansatz calculations. $^{2-6}$ Even the $1 \mathrm{D}, S=\frac{1}{2} X Y$ model, whose thermodynamic properties are those of a system of noninteracting fermions, ${ }^{7,8}$ has highly nontrivial dynamical properties.

The zero-temperature properties of quantum-spin chains are of particular interest, because, at $T=0$, phase transitions occur as a function of various parameters such as an external magnetic field and exchange anisotropies, which are related by rigorous mappings to phase transitions of certain classical models (e.g., Ising and vertex models) as a function of temperature. 9,10

For a number of 1D quantum-spin models such as the $S=\frac{1}{2} X Y Z$ model, the energies of the ground state and of some classes of low-lying excited states are explicitly known. ${ }^{10,11}$ In general, these exact excitation spectra have been found to differ considerably from the "quasiparticle" spectra obtained by many-body perturbation techniques which are standard in 2D and 3D magnetism, ${ }^{12}$ thus providing an indication about the limitations of those approximation techniques for $1 \mathrm{D}$ systems. ${ }^{13}$ Note that these $1 \mathrm{D}$ excitation spectra consist of exact eigenstates of the full Hamiltonian, which therefore have infinite lifetimes, as opposed to the finite lifetimes of quasiparticles. Consequently, they manifest themselves as real (i.e., undamped) singularities in frequency-dependent correlation functions.

In this paper, we shall study the dynamics at $T=0$ of the $1 \mathrm{D}, S=\frac{1}{2}$ transverse Ising (TI) model at the critical external magnetic field and of the $1 \mathrm{D}, S=\frac{1}{2}$ isotropic $X Y$ model in zero field, specified, respectively, by the Hamiltonians

$$
H_{\mathrm{TI}}=-\sum_{l=1}^{N}\left(2 J S_{l}^{x} S_{l+1}^{x}+h_{c} S_{l}^{z}\right), h_{c}=J
$$

and

$$
H_{X Y}=-J \sum_{l=1}^{N}\left(S_{l}^{x} S_{l+1}^{x}+S Y S_{++1}^{y}\right),
$$

in the limit $N \rightarrow \infty$, with periodic boundary conditions imposed. We are interested in the two-spin correlation functions,

$$
\left\langle S_{0}^{\mu}(t) S_{n}^{\mu}\right\rangle=\frac{\operatorname{Tr}\left(e^{-\beta H} e^{i H t} S_{0}^{\mu} e^{-i H t} S_{n}^{\mu}\right)}{\operatorname{Tr}\left(e^{-\beta H}\right)}, \mu=x, y, z
$$

where $\beta=\left(k_{B} T\right)^{-1}$. We use the short-hand notation

$$
\Xi_{n}(t) \equiv 4\left\langle S_{\delta}^{\xi}(t) S_{n}^{\xi}\right\rangle, \quad \xi=x, y, z \text {. }
$$

Valuable information on the excitation spectrum relevant 
for the $T=0$ dynamics is contained in the frequencydependent Fourier transforms,

$$
\Phi_{n}^{\mu \mu}(\omega) \equiv \int_{-\infty}^{+\infty} d t e^{i \omega t}\left\langle S_{0}^{\mu}(t) S_{n}^{\mu}\right\rangle,
$$

and in the dynamic structure factor,

$$
S_{\mu \mu}(q, \omega)=\sum_{n} e^{-i q n} \Phi_{n}^{\mu \mu}(\omega)
$$

The time-dependent correlation functions (1.4) have the following well-known general properties:

$$
\begin{aligned}
& \Xi_{-n}(t)=\Xi_{n}(t), \\
& \Xi_{n}(-t)=\Xi_{n}^{*}(t)=\Xi_{n}(t-i \beta) .
\end{aligned}
$$

Thus, in general, $\Xi_{n}(t)$ is a complex function, except at infinite temperature $(\beta=0)$, where it is real. ${ }^{14}$ The Fouriertransformed functions (1.5) and (1.6), on the other hand, are real functions at any temperature. In fact, both quantities (1.5) and (1.6) are important in the context of dynamical experiments on magnetic compounds exhibiting quasi-1D properties. ${ }^{15} S_{\mu \mu}(q, \omega)$ is directly measured by inelastic neutron scattering, whereas $\Phi_{n}^{\mu \mu}(\omega)$ at small $\omega$, in particular the autocorrelation function (ACF) $\Phi_{0}^{\mu \mu}(\omega)$, is related to the spin-lattice relaxation rate measured by NMR experiments in situations where the relaxation of the NMR probe is dominated by the local fluctuations of the spin chain. ${ }^{16}$

In Fourier space, the symmetry property (1.7b) manifests itself as the well-known detailed balance condition,

$$
\Phi_{n}^{\mu \mu}(-\omega)=e^{-\beta \omega} \Phi_{n}^{\mu \mu}(\omega), \quad S_{\mu \mu}(q,-\omega)=e^{-\beta \omega} S_{\mu \mu}(q, \omega) .
$$

Hence, at $T=0$, both quantities (1.5) and (1.6) vanish identically at negative frequencies. A further useful property of $S_{\mu \mu}(q, \omega)$ is that it is a non-negative function. This property is most easily verified if $S_{\mu \mu}(q, \omega)$ is expressed in the spectral representation,

$$
\begin{gathered}
S_{\mu \mu}(q, \omega)=\frac{2 \pi}{Z} \sum_{\lambda, \lambda^{\prime}} e^{-\beta E_{\lambda}}\left|\left\langle\lambda\left|S^{\mu}(q)\right| \lambda^{\prime}\right\rangle\right|^{2} \\
\times \delta\left(\omega-E_{\lambda}^{\prime}+E_{\lambda}\right) \geq 0,
\end{gathered}
$$

where the sums run over all eigenstates $|\lambda\rangle$ of $H$ with energies $E_{\lambda} ; Z=\sum_{\lambda} e^{-\beta E_{\lambda}}$ is the partition function. The same property holds for the ACF (Ref. 17): $\Phi_{0}^{\mu \mu}(\omega) \geq 0$.

The two Hamiltonians (1.1) and (1.2) are special cases of the more general model,

$$
H=-J \sum_{l=1}^{N}\left[(1+\gamma) S_{l}^{x} S_{l+1}^{x}+(1-\gamma) S S_{l}^{y} S_{+1}^{y}+h S_{l}^{z}\right],
$$

the static properties of which have been studied extensive$1 y .^{7-9,18-20}$ A general formula for the time-dependent correlation function $Z_{n}(t)$ for this model was first derived by Niemeijer. ${ }^{21}$ Various authors have evaluated closedform expressions for $Z_{n}(t)$ and its Fourier transforms for special cases of (1.10). ${ }^{13,22-26}$ In contrast, a complete solution of $X_{n}(t)$ or $Y_{n}(t)$ for (1.10) at arbitrary $T$ has never been found. This can be understood as a result of the fact that, after a Jordan-Wigner transformation from spin operators to fermion operators, $Z_{n}(t)$ involves only a product of four fermion operators, whereas $X_{n}(t)$ and $Y_{n}(t)$ involve an infinite number of such operators. The latter are thus much more complicated objects and represent (in the fermion language) not just two-particle excitations, as is the case for $Z_{n}(t)$, but rather the excitation of arbitrarily many particles. This fact was established by McCoy, Barouch, and Abraham, ${ }^{27}$ who found that $X_{n}(t)$ and $Y_{n}(t)$ for (1.10) can be expressed as infinite block Toeplitz determinants. A complete analysis of these determinants has been given only for infinite temperature. $^{28,29}$ We quote the well-known results ${ }^{28-30}$ for the two models (1.1) and (1.2) at $T=\infty$,

$$
\left[X_{n}(t)\right]_{\mathrm{TI}}=e^{-t^{2} / 2} \delta_{n, 0},\left[X_{n}(t)\right]_{X Y}=e^{-t^{2} / 4} \delta_{n, 0},
$$

where here and henceforth, the units are chosen such that $J \equiv 1$. At $T=0$, the analysis of the Toeplitz determinants was restricted ${ }^{27,31,32}$ to the leading term in the long-time asymptotic expansion (LTAE) of $X_{n}(t)$ and $Y_{n}(t)$ until very recently, when substantial progress was made. ${ }^{33,34}$ The work of Ref. 34 forms the starting point for our present analysis.

A brief report of some of our results was given in Ref. 35. In Sec. II we present a new, extended calculation of the LTAE of the time-dependent ACF's $X_{0}(t)$ and $Y_{0}(t)$ for the TI and $X Y$ models, together with an analysis of their general structural features and a comparison with the ACF's $Z_{0}(t)$. Section III contains a high-precision numerical calculation of the frequency-dependent ACF's $\Phi_{0}^{x x}(\omega)$ and $\Phi_{0}^{y y}(\omega)$ for the TI and $X Y$ models. The singularities of these functions are determined analytically from Fourier transforms of the corresponding LTAE's. These results are discussed in conjunction with similar ones for the frequency-dependent ACF's $\Phi_{0}^{z z}(\omega)$. We define a set of "singularity indices" and comment on the connection between the relative strengths of the singularities and the extent of spin fluctuations in the two models (at $T=0$ ). An appendix contains some details of the calculations of the singularities in the frequency-dependent ACF's.

\section{TIME-DEPENDENT CORRELATION FUNCTIONS}

The time-dependent correlation function $\left[X_{n}(t)\right]_{\mathrm{TI}}$ at $T=0$, can be expressed in terms of a related function $\sigma_{n}(z)$ as $^{34}$

$$
\left[X_{n}(t)\right]_{\mathrm{TI}}=\left[X_{n}(0)\right]_{\mathrm{TI}} \exp \left(-\frac{1}{2} t^{2}+\int_{0}^{2 t} d t^{\prime} \frac{\sigma_{n}\left(i t^{\prime}\right)}{t^{\prime}}\right),
$$

where the static correlation function is given by ${ }^{19,20}$

$$
\left[X_{n}(0)\right]_{\mathrm{TI}}=\left(\frac{2}{\pi}\right)^{|n|} \prod_{l=1}^{|n|}\left(1-\frac{1}{4 l^{2}}\right)^{l-|n|}
$$


and $\sigma_{n}(z)$ satisfies the nonlinear ordinary differential equation (ODE),

$\left(z \sigma_{n}^{\prime \prime}\right)^{2}+4\left(z \sigma_{n}^{\prime}-\sigma_{n}-n^{2}\right)\left[z \sigma_{n}^{\prime}-\sigma_{n}+\left(\sigma_{n}^{\prime}\right)^{2}\right]=0$,

with the initial condition that for $z \rightarrow 0$, the solution can be represented by the series

$$
\sigma_{n}(z)=\sum_{k=1}^{\infty} a_{n, k} z^{2 k}+z^{2 n+1} \sum_{k=0}^{\infty} b_{n, k} z^{2 k}
$$

Here, all coefficients $a_{n, k}$ and $b_{n, k}$ can be calculated recursively in terms of $b_{n, 0}$, where

$$
b_{n, 0}=\left\{\begin{array}{l}
\frac{1}{\pi}, n=0 \\
(-1)^{n} \frac{1}{\pi} \prod_{l=1}^{n}\left[\frac{l}{2(2 l-1)^{2}(2 l+1)}\right], n \geq 1 .
\end{array}\right.
$$

Once $\left[X_{n}(t)\right]_{\mathrm{TI}}$ is known, one can calculate $\left[Y_{n}(t)\right]_{\mathrm{TI}}$ by means of the relation ${ }^{36}$

$$
\left[Y_{n}(t)\right]_{\mathrm{TI}}=-\frac{d^{2}}{d t^{2}}\left[X_{n}(t)\right]_{\mathrm{TI}}
$$

Further, one can compute the correlation functions $\left[X_{n}(t)\right]_{X Y}=\left[Y_{n}(t)\right]_{X Y}$ of the isotropic $X Y$ model (1.2) via the relation ${ }^{28}$

$$
\left[X_{n}(t)\right]_{X Y}=\left[Y_{n}(t)\right]_{X Y}=\left\{\begin{array}{l}
\left\{\left[X_{n / 2}(t / 2)\right]_{\mathrm{TI}}\right\}^{2}, n \text { even } \\
{\left[X_{(n-1) / 2}(t / 2)\right]_{\mathrm{TI}}\left[X_{(n+1) / 2}(t / 2)\right]_{\mathrm{TI}}, n \text { odd }}
\end{array}\right.
$$

Both (2.5) and (2.6) are valid for arbitrary temperature.

For Ref. 34, the ODE (2.2) was solved numerically for $n=0$. From this numerical solution an analytic ansatz for the LTAE of $\sigma_{0}(i t)$ was inferred and then verified analytically. This LTAE was calculated for general $b_{0,0}$ to $O\left(t^{-1}\right)$ and, with some further terms for the physical value $b_{0,0}=1 / \pi$, was used to construct the resultant LTAE of $\left[X_{0}(t)\right]_{\mathrm{TI}}$ to $O\left(t^{-11 / 4}\right)$.
For Ref. 35 the LTAE of $\left[X_{0}(t)\right]_{\text {TI }}$ was extended to the level at which the $e^{-6 i t}$ term (see below) first enters, viz., $O\left(t^{-19 / 4}\right)$. The corresponding LTAE of $\left[Y_{0}(t)\right]_{\mathrm{TI}}$ was also calculated. From an analysis of the general structure of these LTAE's, it was anticipated that terms with the next-higher frequency of oscillation, $e^{-8 i t}$, would first enter at a much more highly suppressed level, namely

\begin{tabular}{|c|c|c|c|c|c|}
\hline$m$ & 0 & 1 & 2 & 3 & 4 \\
\hline$\alpha_{m}^{(x)}$ & 0 & $\frac{1}{2}$ & 2 & $\frac{9}{2}$ & 8 \\
\hline$a_{0}^{(x, m)}$ & 1 & 1 & $\frac{1}{2^{2}}$ & $\frac{1}{2^{5}}$ & $\frac{3}{2^{10}}$ \\
\hline$a_{1}^{(x, m)}$ & 0 & $\frac{9}{2^{3}}$ & $\frac{15}{2^{4}}$ & $\frac{75}{2^{8}}$ & \\
\hline$a_{2}^{(x, m)}$ & $\frac{1}{2^{3}}$ & $\frac{297}{2^{7}}$ & $\frac{489}{2^{7}}$ & $\frac{8881}{2^{12}}$ & \\
\hline$a_{3}^{(x, m)}$ & 0 & $\frac{7587}{2^{10}}$ & $\frac{9387}{2^{9}}$ & $\frac{516609}{2^{15}}$ & \\
\hline$a_{4}^{(x, m)}$ & $\frac{81}{2^{7}}$ & $\frac{1027035}{2^{15}}$ & $\frac{851427}{2^{13}}$ & & \\
\hline$a_{5}^{(x, m)}$ & 0 & $\frac{43594695}{2^{18}}$ & $\frac{22520925}{2^{15}}$ & & \\
\hline$a_{6}^{(x, m)}$ & $\frac{11259}{2^{10}}$ & $\frac{4418168445}{2^{22}}$ & $\frac{1368815805}{2^{18}}$ & & \\
\hline$a_{7}^{(x, m)}$ & 0 & $\frac{260700970635}{2^{25}}$ & & & \\
\hline$a_{8}^{(x, m)}$ & $\frac{13516875}{2^{15}}$ & & & & \\
\hline
\end{tabular}
$O\left(t^{-33 / 4}\right)$. For the present work we have succeeded in

TABLE I. Values for the exponents $\alpha_{m}^{(x)}$ and the coefficients $a_{n}^{(x, m)}$ of the LTAE equation (2.7) of $\left[X_{0}(t)\right]_{\mathrm{TI}}$ which have been calculated. 
TABLE II. Values for the exponents $\alpha_{m}^{(y)}$ and the coefficients $a_{n}^{(y, m)}$ of the LTAE Eq. (2.7) of $\left[Y_{0}(t)\right]_{\mathrm{TI}}$ which have been calculated.

\begin{tabular}{|c|c|c|c|c|c|}
\hline$m$ & 0 & 1 & 2 & 3 & 4 \\
\hline$a_{m}(y)$ & 2 & $\frac{1}{2}$ & 2 & $\frac{9}{2}$ & 8 \\
\hline$a_{\delta}^{(y, m)}$ & $\frac{5}{2^{2}}$ & 4 & 4 & $\frac{9}{2^{3}}$ & $\frac{3}{2^{4}}$ \\
\hline$a_{1}^{(y, m)}$ & 0 & $-\frac{3}{2}$ & 6 & $\frac{447}{2^{6}}$ & \\
\hline$a_{2}^{(y, m)}$ & $\frac{117}{2^{5}}$ & $-\frac{39}{2^{5}}$ & $\frac{315}{2^{4}}$ & $\frac{42025}{2^{10}}$ & \\
\hline$a_{3}^{(y, m)}$ & 0 & $\frac{63}{2^{8}}$ & $\frac{5463}{2^{6}}$ & $\frac{2144637}{2^{13}}$ & \\
\hline$a_{4}^{(y, m)}$ & $\frac{28917}{2^{9}}$ & $-\frac{9765}{2^{13}}$ & $\frac{59373}{2^{7}}$ & & \\
\hline$a_{5}^{(y, m)}$ & 0 & $\frac{136755}{2^{16}}$ & $\frac{6163425}{2^{11}}$ & & \\
\hline$a_{6}^{(y, m)}$ & $\frac{8162775}{2^{12}}$ & $-\frac{12741075}{2^{20}}$ & $\frac{742488885}{2^{15}}$ & & \\
\hline$a^{(y, m)}$ & 0 & $\frac{117223335}{2^{23}}$ & & & \\
\hline$a_{8}^{(y, m)}$ & $\frac{16504104375}{2^{17}}$ & & & & \\
\hline
\end{tabular}

calculating the LTAE's to this level, and, in accord with our expectations, have obtained the leading $e^{-8 i t}$ terms.

We find that the LTAE's of $\left[X_{0}(t)\right]_{\mathrm{TI}}$ and $\left[Y_{0}(t)\right]_{\mathrm{TI}}$ have the following structure, as far as we have determined it:

$$
\begin{aligned}
& {\left[\Xi_{0}(t)\right]_{\mathrm{TI}} \sim \bar{A}(i t)^{-1 / 4} \sum_{m=0}^{\infty} T_{\xi, m}^{(\mathrm{TI})},} \\
& T_{\xi, m}^{(\mathrm{TI})}=(2 \pi)^{-m / 2} e^{-2 i m t}(-2 i t)^{-\alpha_{m}^{(\xi)}} \sum_{n=0}^{\infty} a_{n}^{(\xi, m)}(-2 i t)^{-n},
\end{aligned}
$$

for $\xi=x, y$, where

$$
\bar{A}=2^{1 / 12} \exp \left[3 \xi^{\prime}(-1)\right]=0.64500248 \ldots,
$$

and $\sim$ denotes an asymptotic expansion in standard notation. We have written the LTAE in a form which renders the symmetry property $\Xi_{0}(-t)=\Xi_{0}(t)^{*}$ manifest. The coefficients $a_{n}^{(\xi, m)}$ are rational numbers with $a_{2 n+1}^{(\xi, 0)}=0$ for $\xi=x, y$, and all $n$ in (2.7). (Our present notation for these coefficients differs slightly from that used in Ref. $35 .{ }^{37}$ ) The exponents $\alpha_{m}^{(\xi)}$ are positive integers or half-integers. The values of $a_{n}^{(\xi, m)}$ and $\alpha_{m}^{(\xi)}$ which have been calculated so far are listed in Tables I and II for $\xi=x$ and $y$, respectively. The maximum order in $t^{-1}$ is taken to be $t^{-33 / 4}$ for all $a_{n}^{(\xi, m)}, m=0, \ldots 4$, in accord with the fact that the known term with the highest oscillation frequency enters first at this level, and hence the expansion was calculated to this order of accuracy. It is feasible to calculate the terms with lower oscillation frequencies to higher order; as an example, the $a_{n}^{(x, 0)}$ are listed up to order $t^{-20}$ in Table III.

Some general features of the LTAE (2.7) are as follows.

(i) It consists of an infinite sum of terms $T_{\xi, m}^{(\mathrm{TI})}$, $m=0,1,2, \ldots$, each with a specific oscillatory $t$ dependence given by the phase factor $e^{-2 i m t}$. The fact that all of the terms have frequencies of only one sign is a conse-

TABLE III. Values for the $a_{n}^{(x, 0)}$ for even $n$ from $n=10$ to 20. $\left(a_{n}^{(x, 0)}=0\right.$ for odd $n$. $)$

\begin{tabular}{ll}
\hline \hline$a_{10}^{(x, 0)}$ & $\frac{7093767375}{2^{18}}$ \\
$a_{12}^{(x, 0)}$ & $\frac{11538377839125}{2^{22}}$ \\
$a_{14}^{(x, 0)}$ & $\frac{13449427715059875}{2^{25}}$ \\
$a_{16}^{(x, 0)}$ & $\frac{170204949942527437875}{2^{31}}$ \\
$a_{18}^{(x, 0)}$ & $\frac{350982198204787691206875}{2^{34}}$ \\
$a_{20}^{(x, 0)}$ & $\frac{1829524473982512079025439375}{2^{38}}$ \\
\hline
\end{tabular}


quence of the condition of detailed balance [Eq. (1.8)], which implies that at $T=0, \phi \xi^{\xi}(\omega)=0$ for $\omega<0$.

(ii) Each term $T_{\xi, m}^{(\mathrm{TI})}$ is, itself, an infinite sum of terms with ascending powers of $t^{-1}$. We thus denote it as a "tower." Each successive tower enters first at a progressively higher level in the expansion; that is, the higher the oscillation frequency, the more highly suppressed the tower is in $t$. Our results in Table I strongly suggest that the exponent $\alpha_{m}^{(x)}$ governing the degree of this suppression, is given by $\alpha_{m}^{(x)}=m^{2} / 2$. From (2.5), it follows that $\alpha_{m}^{(y)}=m^{2} / 2$ also, except for the nonoscillatory tower, which is suppressed by two units ${ }^{38}: \alpha_{0}^{(y)}=2$. This is reflected in Table II.

(iii) The dominant term in $\left[X_{0}(t)\right]_{\mathrm{TI}}$ for large $t$ is $\bar{A}(i t)^{-1 / 4}$, which is nonoscillatory and reflects Lorentz invariance in the scaling limit ${ }^{39}$ given the result ${ }^{20}$ that $\left[X_{n}(0)\right]_{\mathrm{TI}} \sim \bar{A} n^{-1 / 4}$ for large $n$. In contrast, the dominant term in $\left[Y_{0}(t)\right]_{\mathrm{TI}}$ for large $t$ is $2 \bar{A}(i t)^{-1 / 4}(-i t)^{-1 / 2} e^{-2 i t}$, which is oscillatory in character and does not connect via Wick rotation with the leading term of the static correlation function ${ }^{20}$ for large $n,\left[Y_{n}(0)\right]_{\mathrm{TI}} \sim(5 \bar{A} / 16) n^{-9 / 4}$. Note, however, that the term $(5 \bar{A} / 16)(i t)^{-9 / 4}$ does appear in the LTAE of $\left[Y_{0}(t)\right]_{\mathrm{TI}}$ and is the leading term of the nonoscillatory tower, but is not the overall leading term in this function.

By using (2.6), we have also calculated the LTAE of $\left[X_{0}(t)\right]_{X Y}=\left[Y_{0}(t)\right]_{X Y}$ from (2.7) and have found the following result ( $\perp$ denotes $x$ or $y$ ):
$\left[X_{0}(t)\right]_{X Y} \sim(\bar{A})^{2} 2^{1 / 2}(i t)^{-1 / 2} \sum_{m=0}^{\infty} T_{\perp, m}^{(X Y)}$

$T_{\perp, m}^{(X Y)}=(2 \pi)^{-m / 2} e^{-i m t}(-i t)^{-\beta_{m}^{(\perp)}} \sum_{n=0}^{\infty} b_{n}^{(1, m)}(-i t)^{-n}$

The coefficients $b_{n}^{(\perp, m)}$ are positive rational numbers, with $b_{2 n+1}^{(\perp, 0)}=0$ for all $n$ in (2.9), and the exponents $\beta_{m}^{(\perp)}$ are positive integers or half-integers. ${ }^{37}$ Table IV lists the values which have been calculated. Evidently, the structure of (2.9) is very similar to that of (2.7). In contrast to the TI case, however, all non-negative integral frequencies, not only the even ones, occur in the LTAE of $\left[X_{0}(t)\right]_{X Y}$. The leading term in $(2.9),(\bar{A})^{2} 2^{1 / 2}(i t)^{-1 / 2}$, can again be understood as reflecting Lorentz invariance in a scaling limit, given the result ${ }^{18}$ that $\left[X_{n}(0)\right]_{X Y} \sim n^{-1 / 2}$ for large $n$. As before, the higher-frequency towers enter at more highly suppressed levels in $t$; in this case our results suggest that the exponent in the prefactor for the $m$ th tower is given by $\beta_{m}^{(1)}=\frac{1}{2}\left[\left(m^{2}+1\right) / 2\right]$, where $[v]$ denotes the integer part of $v$.

It is interesting to compare these new results for $X_{0}(t)$ and $Y_{0}(t)$ with the known results for $Z_{0}(t)$. In fact, Niemeijer's expression ${ }^{21}$ for $Z_{n}(t)$ in the general model (1.10) can be evaluated for the special cases (1.1) and (1.2) at $T=0$, in terms of Bessel functions $J_{n}$ and Weber functions $E_{n}$. The results are

TABLE IV. Values for the exponents $\beta_{m}^{(\perp)}$ and the coefficients $b_{n}^{(\perp, m)}$ of the LTAE equation (2.9) of $\left[X_{0}(t)\right]_{X Y}=\left[Y_{0}(t)\right]_{X Y}$ which have been calculated.

\begin{tabular}{|c|c|c|c|c|c|}
\hline$m$ & 0 & 1 & 2 & 3 & 4 \\
\hline$\beta_{m}^{(1)}$ & 0 & $\frac{1}{2}$ & 1 & $\frac{5}{2}$ & 4 \\
\hline$b_{0}^{(1, m)}$ & 1 & 2 & 1 & $\frac{1}{2}$ & $\frac{1}{2^{4}}$ \\
\hline$b_{1}^{(1, m)}$ & 0 & $\frac{9}{2^{2}}$ & $\frac{11}{2^{2}}$ & $\frac{39}{2^{4}}$ & $\frac{17}{2^{5}}$ \\
\hline$b_{2}^{(\perp, m)}$ & $\frac{1}{2^{2}}$ & $\frac{313}{2^{6}}$ & $\frac{249}{2^{5}}$ & $\frac{2809}{2^{8}}$ & $\frac{441}{2^{7}}$ \\
\hline$b_{3}^{(1, m)}$ & 0 & $\frac{7731}{2^{9}}$ & $\frac{3551}{2^{7}}$ & $\frac{110397}{2^{11}}$ & $\frac{10993}{2^{9}}$ \\
\hline$b_{4}^{(1, m)}$ & $\frac{41}{2^{5}}$ & $\frac{1057275}{2^{14}}$ & $\frac{249123}{2^{11}}$ & $\frac{19710331}{2^{16}}$ & $\frac{285033}{2^{11}}$ \\
\hline$b_{5}^{(1, m)}$ & 0 & $\frac{44024103}{2^{17}}$ & $\frac{5297313}{2^{13}}$ & $\frac{1008538089}{2^{19}}$ & \\
\hline$b_{6}^{(1, m)}$ & $\frac{2835}{2^{7}}$ & $\frac{4486876461}{2^{21}}$ & $\frac{266181453}{2^{16}}$ & & \\
\hline$b_{7}^{(\perp, m)}$ & 0 & $\frac{261970861563}{2^{24}}$ & $\frac{7765967691}{2^{18}}$ & & \\
\hline$b_{8}^{(1, m)}$ & $\frac{1696059}{2^{11}}$ & & & & \\
\hline
\end{tabular}




$$
\begin{aligned}
{\left[Z_{n}(t)\right]_{\mathrm{TI}}=} & \frac{4}{\pi^{2}}+\left[J_{2 n}(2 t)+i E_{2 n}(2 t)\right]^{2} \\
& -\left[J_{2 n-1}(2 t)+i E_{2 n-1}(2 t)\right] \\
& \times\left[J_{2 n+1}(2 t)+i E_{2 n+1}(2 t)\right]
\end{aligned}
$$

and

$$
\left[Z_{n}(t)\right]_{X Y}=\left[J_{n}(t)+i E_{n}(t)\right]^{2}
$$

respectively. ${ }^{40}$ By using standard mathematical references, ${ }^{41}$ one can calculate the LTAE's of $Z_{n}(t)$ to all orders. We find that they have a structure which is similar to (2.7) and (2.9) but contains only three towers. The results for $n=0$ are

$$
\begin{aligned}
& {\left[Z_{0}(t)\right]_{\mathrm{TI}}-\frac{4}{\pi^{2}} \sim \sum_{m=0}^{2} T_{z, m}^{(\mathrm{TI})},} \\
& T_{z, m}^{(\mathrm{TI})}=\pi^{(m / 2)-2} e^{-2 i m t}(-2 i t)^{-\alpha_{m}^{(z)}} \sum_{n=0}^{\infty} a_{n}^{(z, m)}(-2 i t)^{-n},
\end{aligned}
$$

with $\alpha_{0}^{(z)}=2, \alpha_{1}^{(z)}=\frac{3}{2}, \alpha_{2}^{(z)}=2$, and rational coefficients $a_{n}^{(z, m)} ;$ and

$$
\begin{aligned}
& {\left[Z_{0}(t)\right]_{X Y} \sim \sum_{m=0}^{2} T_{z, m}^{(X Y)},} \\
& T_{z, m}^{(X Y)}=\pi^{(m / 2)-2} e^{-i m t}(-i t)^{-\beta_{m}^{(z)}} \sum_{n=0}^{\infty} b_{n}^{(z, m)}(-i t)^{-n},
\end{aligned}
$$

with $\beta_{0}^{(z)}=2, \beta_{1}^{(z)}=\frac{3}{2}, \beta_{2}^{(z)}=1$, and rational coefficients $b_{n}^{(z, m)}$. The leading term of (2.12a) comes from the second tower $(m=1)$ and is

$$
2^{5 / 2} \pi^{-3 / 2} e^{-2 i t}(-2 i t)^{-3 / 2}
$$

whereas the leading term of (2.13a) comes from the third tower $(m=2)$ and is

$$
2 \pi^{-1} e^{-2 i t}(-i t)^{-1}
$$

The fact that $\left[X_{0}(t)\right]_{\mathrm{TI}}$ falls off less rapidly than both $\left[Y_{0}(t)\right]_{\mathrm{TI}}$ and $\left[Z_{0}(t)\right]_{\mathrm{TI}}$ at large $t$ reflects the property that the $x$ axis is the "easy" spin-fluctuation direction. More specifically, for $h<h_{c}=1$, there is a net magnetization $M_{x}=\frac{1}{2}\left[\lim _{n \rightarrow \infty} X_{n}(0)\right]^{1 / 2}$ in the TI model. As $h$ increases through $h_{c}$, this long-range order disappears. At the critical value $h_{c}=1$, there are thus large fluctuations in the order parameter $\boldsymbol{M}_{\boldsymbol{x}}$. By the Lorentz invariance correspondence noted above in the scaling limit, these strong fluctuations in the static correlation function $\left[X_{n}(0)\right]_{\mathrm{TI}}$ for large $n$ are equivalent to strong fluctuations governing $\left[X_{0}(t)\right]_{\mathrm{TI}}$ at large $t$. By analogy, the fact that $\left[Z_{0}(t)\right]_{X Y}$ falls off more rapidly than $\left[X_{0}(t)\right]_{X Y}=\left[Y_{0}(t)\right]_{X Y}$ reflects the property that the $x y$ plane is the easy plane for spin fluctuations in the $X Y$ model. ${ }^{18,42}$

The time-dependent correlation functions $\left[X_{n}(t)\right]_{X Y}$ and $\left[Z_{n}(t)\right]_{X Y}$ were studied ${ }^{39,43}$ in the framework of the Luttinger model, an exactly solvable Fermi-field theory in $1+1$ dimensions. ${ }^{44}$ The Luttinger model is understood to represent a continuum version of the $1 \mathrm{D}, S=\frac{1}{2}, X X Z$ model which contains the $X Y$ model (1.2) as a special case, namely that of free fermions. The following results have been found for the ACF's of the Luttinger model in the free-fermion limit ${ }^{39,43}$ :

$$
\left[X_{0}(t)\right]_{\mathrm{Lut}} \sim C_{1} t^{-1 / 2}+C_{2} t^{-5 / 2}
$$

and

$$
\left[Z_{0}(t)\right]_{\mathrm{Lut}} \sim C_{3} t^{-2},
$$

where the $C_{j}$ depend on the cutoff parameters and scaling variables. Comparison with the exact LTAE's (2.9) and (2.13) of the $X Y$ model makes it clear that a great deal of information on the dynamics of the quantum-spin model (1.2) is lost if the calculation is done in the continuum limit. The Luttinger-model calculation reproduces the correct exponents in the first two terms of the nonoscillatory tower of $\left[X_{0}(t)\right]_{X Y}$ and in the first term of the nonoscillatory tower of $\left[Z_{0}(t)\right]_{X Y}$, but gives no further nonoscillatory terms. It fails to produce any of the oscillatory terms. This is particularly serious in the case of $\left[Z_{0}(t)\right]_{X Y}$, where the oscillatory towers include the leading and the next-leading term of the LTAE.

A final general feature of all of these correlation functions concerns the effect of the spin-spin interaction strength. The basic observation is that the correlation functions are (for $\hbar=1$ ) dimensionless functions of $J$ and $t$, and therefore can only depend on these variables in the single combination $J t$. (This is implicit in our notation, where $J=1$.) Hence, it follows, in agreement with one's intuition, that the larger $J$ is, the more rapidly the spinspin correlations approach their asymptotic values.

In addition to determining the analytic long-time asymptotic expansions of $\left[X_{0}(t)\right]_{\mathrm{TI}}, \quad\left[Y_{0}(t)\right]_{\mathrm{TI}}$, and $\left[X_{0}(t)\right]_{X Y}=\left[Y_{0}(t)\right]_{X Y}$, it is quite useful to calculate these functions numerically for general $t$. For this purpose we have solved the ODE (2.2) numerically and have determined $\left[X_{0}(t)\right]_{\mathrm{TI}}$ according to $(2.1){ }^{45}\left[Y_{0}(t)\right]_{\mathrm{TI}}$ is then calculated by numerical differentiation according to (2.5), and $\left[X_{0}(t)\right]_{X Y}=\left[Y_{0}(t)\right]_{X Y}$ is obtained from (2.6). The results are displayed in Figs. 1 and 2. For comparison, we also show $Z_{0}(t)$ as given for the two models by (2.10) and (2.11). The property (1.7b) implies that

$$
\operatorname{Im}\left[\Xi_{0}(t=0)\right]=0, \quad \Xi=X, Y, Z
$$

(at arbitrary $T$ ), and this is evident in all of the plots. This condition (1.7b), in conjunction with the fact that the correlation functions are entire functions of $t,^{28}$ implies that

$$
\left.\frac{d}{d t} \operatorname{Re}\left[\Xi_{0}(t)\right]\right|_{t=0}=0, \quad \Xi=X, Y, Z
$$



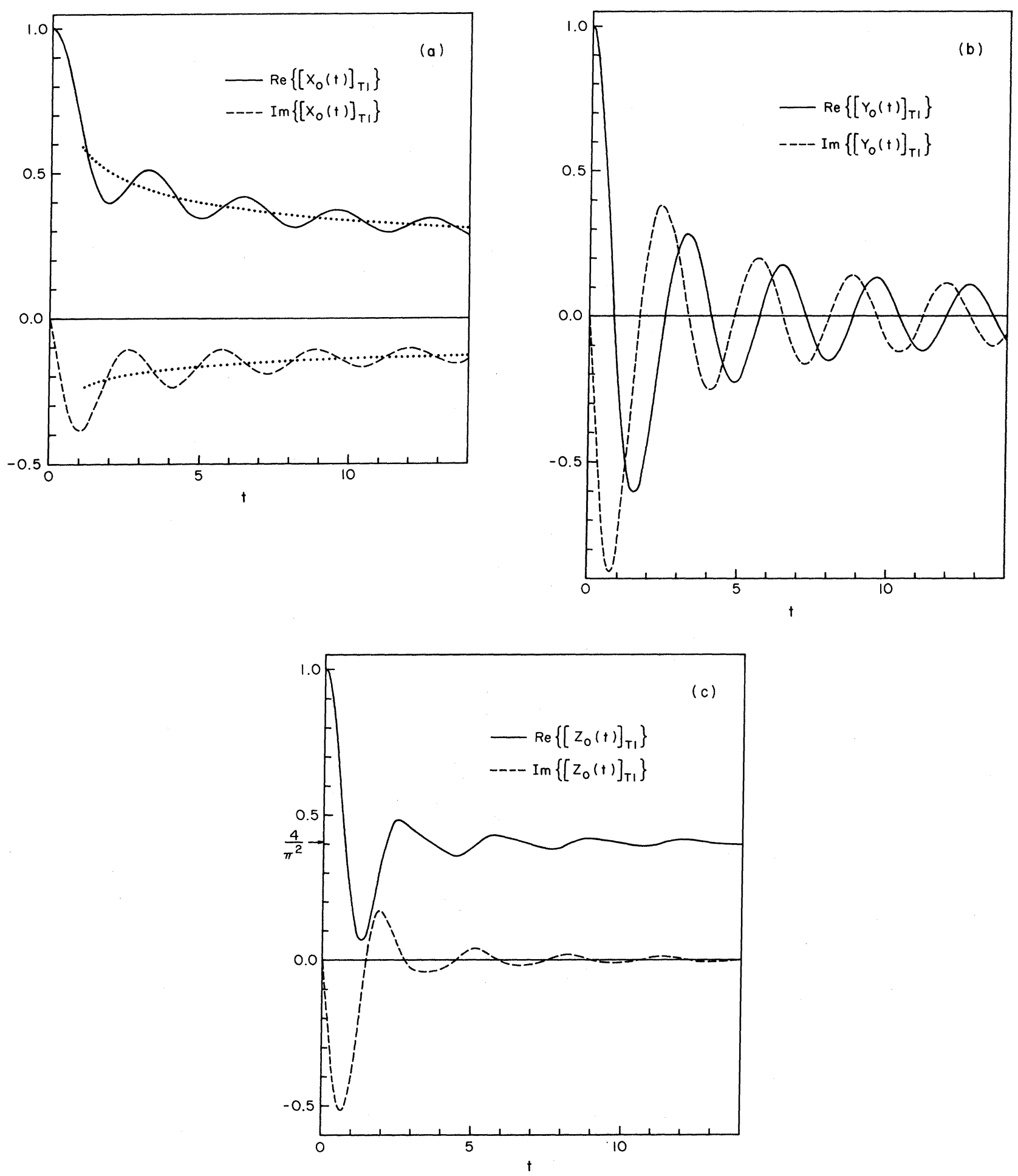

FIG. 1. Short-time behavior of time-dependent ACF's: (a) $\left[X_{0}(t)\right]_{\mathrm{TI}},(\mathrm{b})\left[Y_{0}(t)\right]_{\mathrm{TI}}$, and (c) $\left[Z_{0}(t)\right]_{\mathrm{TI}}$. Solid and dashed lines denote, respectively, the real and imaginary part of the ACF's. Dotted lines in (a) represent real and imaginary parts of the leading, nonoscillatory term of the LTAE of $\left[X_{0}(t)\right]_{\mathrm{TI}}$. The real part of $\left[Z_{0}(t)\right]_{\mathrm{TI}}$ approaches the value $4 / \pi^{2}$ asymptotically in the limit $t \rightarrow \infty$.

and again, this is clear in all of the graphs. We also note that $^{34}$

$$
\frac{d}{d t} \operatorname{Im}\left\{\left[X_{0}(t)\right]_{\mathrm{TI}}\right\}=-2 M_{z},
$$

where $M_{z}$ is the magnetization of the TI model. At $T=0$, this is ${ }^{7,8} M_{z}=1 / \pi$. Similar comments apply for the time derivatives of the other ACF's at $t=0$.

The most prominent features of the long-time asymp- 

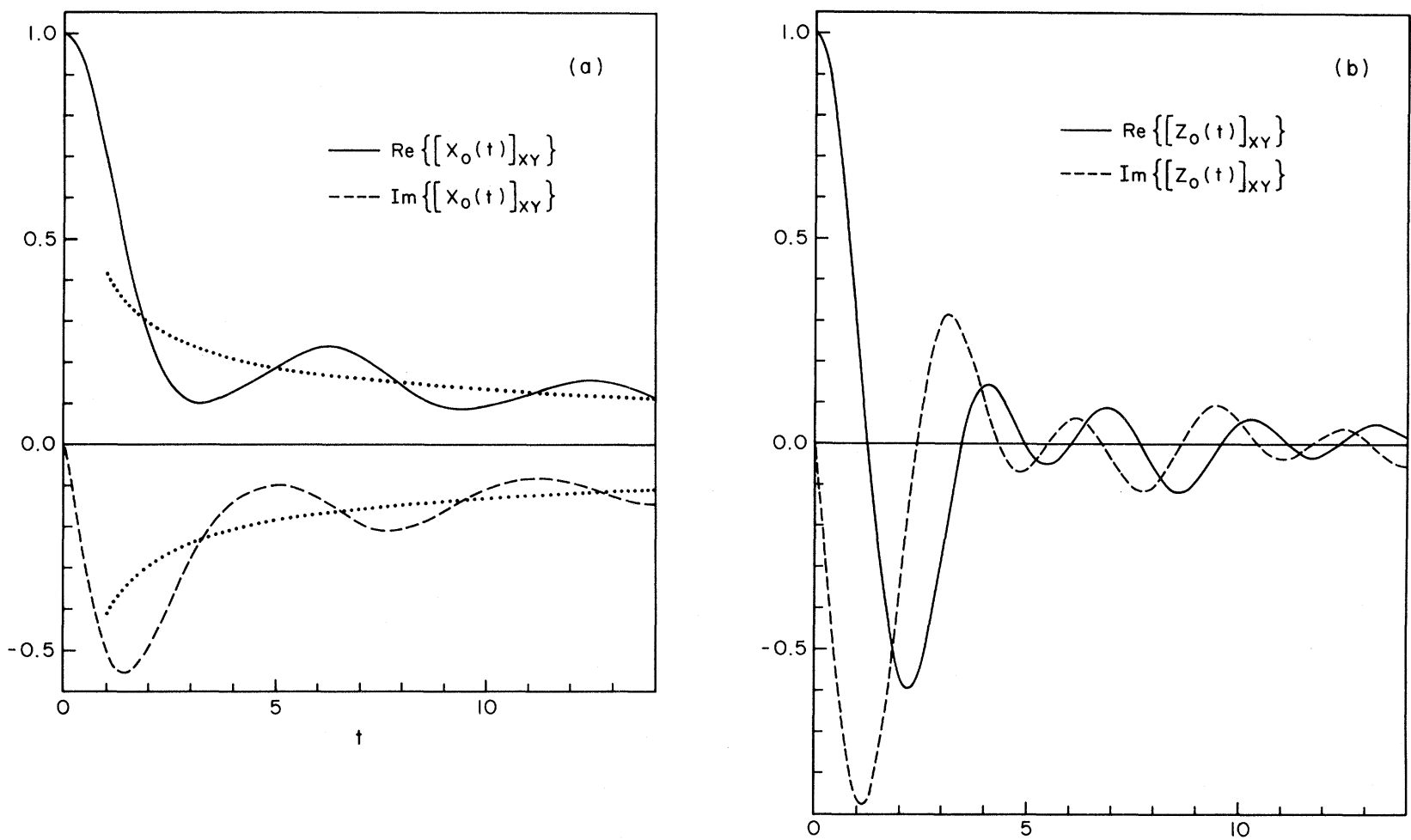

FIG. 2. Short-time behavior of time-dependent ACF's: (a) $\left[X_{0}(t)\right]_{X Y}=\left[Y_{0}(t)\right]_{X Y}$ and (b) $\left[Z_{0}(t)\right]_{X Y}$. Solid and dashed lines denote, respectively, the real and imaginary part of the ACF's. Dotted lines in (a) represent real and imaginary parts of the leading, nonoscillatory term of the LTAE of $\left[X_{0}(t)\right]_{X Y}$.

totic behavior discussed above can already be discerned for short times. As is evident in Fig. 1(a), the graphs of $\operatorname{Re}\left\{\left[X_{0}(t)\right]_{\mathrm{TI}}\right\}$ and $\operatorname{Im}\left\{\left[X_{0}(t)\right]_{\mathrm{TI}}\right\}$ execute oscillations around the uniform curves $\bar{A} \cos (\pi / 8) t^{-1 / 4}$ and $-\bar{A} \sin (\pi / 8) t^{-1 / 4}$, which approach zero monotonically as $t \rightarrow \infty$. The behavior of $\left[X_{0}(t)\right]_{X Y}=\left[Y_{0}(t)\right]_{X Y}$ is similar, but the real and imaginary parts oscillate around the more rapidly dying curves $\pm(\bar{A})^{2} t^{-1 / 2}$, respectively. In contrast, $\left[Y_{0}(t)\right]_{\mathrm{TI}}$ and $\left[Z_{0}(t)\right]_{X Y}$ fall off as $t \rightarrow \infty$ via damped oscillations around zero. In the same limit, $\left[Z_{0}(t)\right]_{\mathrm{TI}}$ approaches a nonzero asymptotic value, $\left[Z_{0}(\infty)\right]_{\mathrm{TI}}=4 M_{z}^{2}=4 / \pi^{2}$. Both $\operatorname{Re}\left\{\left[Z_{0}(t)\right]_{\mathrm{TI}}\right\}-4 / \pi^{2}$ and $\operatorname{Im}\left\{\left[Z_{0}(t)\right]_{\mathrm{TI}}\right\}$ die off with damped oscillations about zero as $t \rightarrow \infty$.

\section{FREQUENCY-DEPENDENT CORRELATION FUNCTIONS}

We have generated the function $\left[\Phi_{0}^{x x}(\omega)\right]_{\mathrm{TI}}$ numerically by a fast-Fourier-transform program using the precise numerical results for $\left[X_{0}(t)\right]_{\mathrm{TI}}$ for $t<40$ and the LTAE (2.7) for $t>40$. At $t=40$, our numerical solution matches the known LTAE to within an error of $10^{-6}$. The resulting $\left[\Phi_{0}^{x x}(\omega)\right]_{\mathrm{TI}}$ is plotted in Fig. 3. The accuracy of the curve is estimated to be better than one part in $10^{3}$ over the range of $\omega$ shown. Figure 3 also shows $\left[\Phi_{0}^{y y}(\omega)\right]_{\mathrm{TI}}$, which is, according to (2.5), given by

$$
\left[\Phi_{0}^{y y}(\omega)\right]_{\mathrm{TI}}=\omega^{2}\left[\Phi_{0}^{x x}(\omega)\right]_{\mathrm{TI}} \text {. }
$$

These frequency-dependent ACF's have quite striking behavior. The results shown in Fig. 3 exhibit singularities at $\omega=0,2$, and 4 . $\left[\Phi_{0}^{x x}(\omega)\right]_{\mathrm{TI}}$ has divergences at $\omega=0$ and 2 and infinite curvature at $\omega=4$. It is a rigorous property that the singularities in the frequency-dependent correlation functions are determined by the long-time asymptotic behavior of the corresponding time-dependent correlation functions. By using our result (2.7) on the LTAE of $\left[X_{0}(t)\right]_{\mathrm{TI}}$, we find that $\left[\Phi_{0}^{x x}(\omega)\right]_{\mathrm{TI}}$ has further (finite) singularities at $\omega=6,8, \ldots$, dominated by the leading term in each successive tower of the LTAE. Specifically, the dominant singularity of $\left[\Phi_{0}^{x x}(\omega)\right]_{\mathrm{TI}}$ at frequency

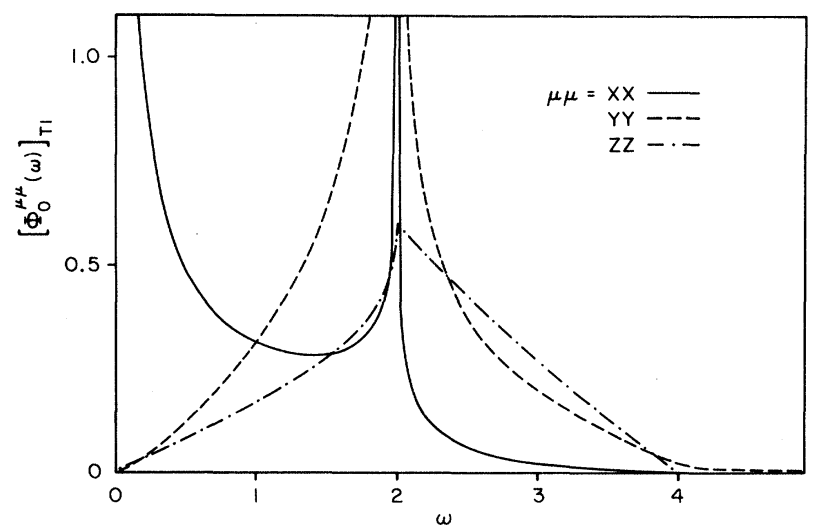

FIG. 3. Frequency-dependent ACF's $\left[\Phi_{0}^{x x}(\omega)\right]_{\mathrm{TI}}$ (solid line), $\left[\Phi_{0}^{y y}(\omega)\right]_{\mathrm{TI}}$ (dashed line), and $\left[\Phi_{0}^{z z}(\omega)\right]_{\mathrm{TI}}$ (dotted-dashed line). 
$\omega_{m}^{(\mathrm{TI})}=2 m, m=0,1,2, \ldots$, has the following form:

$$
\begin{aligned}
{\left[\Phi_{0}^{x x}(\omega)\right]_{\mathrm{TI}}^{(m)} \sim } & \frac{1}{2} A_{m}^{(x)} \Gamma\left(\frac{3}{4}-\frac{1}{2} m^{2}\right)|\omega-2 m|^{v_{x, m}^{(\mathrm{TI})}} \\
\times & \left\{\frac{1}{2}\left[1-(-1)^{m}\right] \theta(2 m-\omega)\right. \\
& \left.+2^{-1 / 2} \theta(\omega-2 m)\right\}
\end{aligned}
$$

where

$$
A_{m}^{(x)}=\bar{A}(2 \pi)^{-m / 2} 2^{-m^{2} / 2} a_{0}^{(x, m)}
$$

and

$$
v_{x, m}^{(\mathrm{TI})}=\frac{m^{2}}{2}-\frac{3}{4},
$$

From Table I we can determine the amplitudes $A_{m}^{(x)}$ explicitly up to $m=4$. Our calculational method is described in the Appendix. Note that the power-type singularities in $\left[\Phi_{0}^{x x}(\omega)\right]_{\text {TI }}$ at $\omega=\omega_{m}^{(\mathrm{TI})}$ are one sided for even $m$ and two sided for odd $m$. Two of the singularities are divergent, viz., $\sim \omega^{-3 / 4} \theta(\omega)$ at $\omega \simeq 0$ and $\sim|\omega-2|^{-1 / 4}[\theta(2-\omega)$ $\left.+2^{-1 / 2} \theta(\omega-2)\right]$, at $\omega \simeq 2$. The "singularity exponents" $v_{x, m}^{(\mathrm{TI})}$ measure the strength of the singularities. Since $v_{x, m}^{(\mathrm{TI})}$ is a monotonically increasing function of $m$, the singularities become progressively weaker for larger $m$. The maximum allowed strength of a singularity in a frequencydependent correlation function, i.e., the lower bound on a general singularity exponent $v$ is $v>-1$. This follows from the physical requirement that the integral

$$
\int_{0}^{\infty} \frac{d \omega}{2 \pi} \Phi_{0}^{\mu \mu}(\omega)=\left\langle\left(S^{\mu}\right)^{2}\right\rangle
$$

be finite. Parenthetically, the value $v=-1$ is approached in the limit of infinite spin dimensionality $S$ in the 1D, isotropic Heisenberg (HB) model at $T=0$, where w $^{46-48}$

$$
v_{\xi, 0}^{(\mathrm{HB})}=-1+2 /(\pi S)+O\left(S^{-2}\right), \quad \xi=x, y, z .
$$

The dominant singularities in $\left[\Phi_{0}^{y y}(\omega)\right]_{\mathrm{TI}}$ are

$$
\left[\Phi_{0}^{y y}(\omega)\right]_{\mathrm{TI}}^{(m)} \sim\left\{\begin{array}{l}
\omega^{2} \Phi_{0}^{x x}(\omega)_{\mathrm{TI}}^{(0)}, \quad m=0 \\
(2 m)^{2} \Phi_{0}^{x x}(\omega)_{\mathrm{TI}}^{(m)}, \quad m \neq 0
\end{array}\right.
$$

$$
\left[\Phi_{0}^{\perp \perp}(\omega)\right]_{X Y}^{(m)} \sim\left\{\begin{array}{l}
\frac{1}{2} B_{m}^{(\perp)} \Gamma\left(\frac{1}{2}-\frac{1}{4} m^{2}\right)(\omega-m)^{v_{\perp, m}^{(X Y)}} \theta(\omega-m), \text { even } m \\
-\frac{1}{2} B_{m}^{(\perp)} \frac{1}{\left[\frac{1}{4}\left(m^{2}-1\right)\right] !}|\omega-m|^{v_{\perp, m}^{(X Y)} \ln |\omega-m|, \text { odd } m}
\end{array}\right.
$$

where

$$
B_{m}^{(\perp)}=(\bar{A})^{2} 2^{1 / 2}(2 \pi)^{-m / 2} b_{0}^{(\perp, m)},
$$

and

$$
v_{\perp, m}^{(X Y)}= \begin{cases}\frac{m^{2}}{4}-\frac{1}{2}, & m \text { even } \\ \frac{m^{2}}{4}-\frac{1}{4}, & m \text { odd }\end{cases}
$$


The $B_{m}^{(\perp)}$ can be determined explicitly up to $m=4$ from the values of $b_{0}^{(\perp, m)}$ given in Table IV. The singularities in $\left[\Phi_{0}^{\perp 1}(\omega)\right]_{X Y}$ at $\omega_{m}^{(X Y)}$ are alternatingly one-sided power type ( $m$ even) and two-sided power type with logarithmic corrections $\left(m\right.$ odd). In particular, the singularities shown in Fig. 4 are the two divergences $\sim \omega^{-1 / 2} \theta(\omega)$ at $\omega \simeq 0$ and $\sim \ln |\omega-1|$ at $\omega \simeq 1$, and the cusp $\sim(\omega-2)^{1 / 2} \theta(\omega-2)$ at $\omega \simeq 2$. Note that each of the two divergent singularities in $\left[\Phi_{0}^{\perp \perp}(\omega)\right]_{X Y}$ is weaker by $\frac{1}{4}$ of a unit in $v$ than the respective singularity in $\left[\Phi_{0}^{x x}(\omega)\right]_{\mathrm{TI}}$.

Our numerical results show that in both the TI and $X Y$ models $\Phi \xi \xi(\omega), \xi=x, y$, approach zero very rapidly as $\omega$ increases beyond 2. Actually, the large- $\omega$ behavior of these frequency-dependent ACF's is strongly constrained by the property that the time-dependent ACF's are entire functions of $t{ }^{28}$ As a result of this property, $\Phi{ }^{\xi \xi}(\omega), \xi=x, y$, must vanish at least as rapidly as $\sim \exp \left(-a \omega^{2}\right)$ where $a>0$, as $\omega \rightarrow \infty . .^{49,50}$

For a physical interpretation of $\left[\Phi_{0}^{x x}(\omega)\right]_{\mathrm{TI}},\left[\Phi_{0}^{y y}(\omega)\right]_{\mathrm{TI}}$, and $\left[\Phi_{0}^{\perp \perp}(\omega)\right]_{X Y}$, it is instructive to compare these functions with $\left[\Phi_{0}^{z z}(\omega)\right]_{\mathrm{TI}}$ and $\left[\Phi_{0}^{z z}(\omega)\right]_{X Y}$, both of which can be evaluated exactly in terms of elliptic integrals: ${ }^{41}$

$$
\left[\Phi_{0}^{z z}(\omega)\right]_{\mathrm{TI}}=\frac{2}{\pi}[K(k)-E(k)] \theta(\omega) \theta(4-\omega)+V(\omega) \theta(\omega) \theta(2-\omega)
$$

with

$$
\begin{aligned}
& V(\omega)=\frac{2}{\pi}\left\{F\left(\gamma_{1}, k\right)-F\left(\gamma_{2}, k\right)-E\left(\gamma_{1}, k\right)+E\left(\gamma_{2}, k\right)+\tan (u)\left[\cos ^{2} u-(\omega / 4)^{2}\right]^{1 / 2}-\cot (u)\left[\sin ^{2} u-(\omega / 4)^{2}\right]^{1 / 2}\right\}, \\
& \gamma_{1}=\arcsin [\sin (u) / k], \quad \gamma_{2}=\arcsin [\cos (u) / k] \\
& k=\left[1-(\omega / 4)^{2}\right]^{1 / 2}, \sin (2 u)=\omega / 2
\end{aligned}
$$

and

$$
\begin{aligned}
{\left[\Phi_{0}^{z z}(\omega)\right]_{X Y}=} & \frac{4}{\pi} F\left[\arcsin \left(\left\{\frac{1}{2}\left[1-\left(1-\omega^{2}\right)^{1 / 2}\right]\right\}^{1 / 2} / k\right),\left[1-(\omega / 2)^{2}\right]^{1 / 2}\right] \theta(\omega) \theta(1-\omega) \\
& +\frac{2}{\pi} K\left\{\left[1-(\omega / 2)^{2}\right]^{1 / 2}\right\} \theta(\omega-1) \theta(2-\omega)
\end{aligned}
$$

These functions are also plotted in Figs. 3 and 4 . In contrast to the behavior of $\Phi_{0}^{x x}(\omega)$ and $\Phi_{0}^{y y}(\omega)$, which have an infinite number of singularities, the functions $\Phi_{0}^{z z}(\omega)$ have only three singularities corresponding to the presence of only three towers in the LTAE's (2.12) and (2.13) of $Z_{0}(t)$. Specifically, the dominant singularities of $\left[\Phi_{0}^{z z}(\omega)\right]_{\mathrm{TI}}$ at $\omega=2 m, m=0,1,2$, are, respectively,

$$
\left[\Phi_{0}^{\mathrm{zz}}(\omega)\right]_{\mathrm{TI}, c}^{(m)} \sim\left\{\begin{array}{l}
(\omega / 2 \pi) \theta(\omega) \\
-(2 / \pi)(2-\omega)^{1 / 2} \theta(2-\omega), \\
\frac{1}{4}(4-\omega) \theta(4-\omega)
\end{array}\right.
$$

where $\left[\Phi_{0}^{z z}(\omega)\right]_{\mathrm{TI}, c}=\left[\Phi_{0}^{z z}(\omega)\right]_{\mathrm{TI}}-M_{z}^{2} \delta(\omega)$, and the dominant singularities or $\left[\Phi_{0}^{\mathrm{zz}}(\omega)\right]_{X Y}$ at $\omega=m, m=0,1,2$, have, respectively, the following forms:

$$
\Phi_{0}^{\underline{Z Z}}(\omega)_{X Y}^{(m)} \sim\left\{\begin{array}{l}
(2 / \pi) \omega \theta(\omega) \\
-2^{5 / 2} \pi^{-1}(1-\omega)^{1 / 2} \theta(1-\omega) . \\
\theta(2-\omega)
\end{array}\right.
$$

Thus, the singularity exponents are $v_{z, m}^{(\mathrm{TI})}=1, \frac{1}{2}, 1$; and $v_{z, m}^{(X Y)}=1, \frac{1}{2}, 0$ for $m=0,1,2$, respectively. Note that none of the singularities in $\phi_{0}^{z z}(\omega)$ is divergent. ${ }^{51}$ A closely related difference in behavior is that the functions $\left[\phi_{0}^{z z}(\omega)\right]_{\mathrm{TI}}$ and $\left[\phi_{0}^{z z}(\omega)\right]_{X Y}$ are nonzero only for $0<\omega<4$ and $0<\omega<2$, respectively, whereas $\phi_{0}^{x x}(\omega)$ and $\phi_{0}^{y y}(\omega)$ have spectral weight at arbitrarily high frequencies. This difference in behavior can be understood in the framework of the fermion representation of the models (1.1) and (1.2) or the more general model (1.10); the ground state is characterized by a half-filled band of noninteracting, spinless fermions with one-particle energies,

$$
\left[\epsilon_{1}(q)\right]_{\mathrm{TI}}=2 \cos \frac{q}{2},\left[\epsilon_{1}(q)\right]_{X Y}=\cos q .
$$

Now, the spin operator $S_{l}^{z}$, if applied to the ground state, couples to the two-particle excitations only, whereas the operators $S_{l}^{x}$ and $S_{l}^{y}$ couple to $m$-particle excitations with $m$ arbitrarily large. ${ }^{27}$ Consequently, the two-particle spectrum exhausts all spectral weight in $\Phi_{0}^{z z}(\omega)$, whereas $\Phi_{0}^{x x}(\omega)$ and $\Phi_{0}^{y y}(\omega)$ include also contributions from $m$ particle excitations with $m>2$.

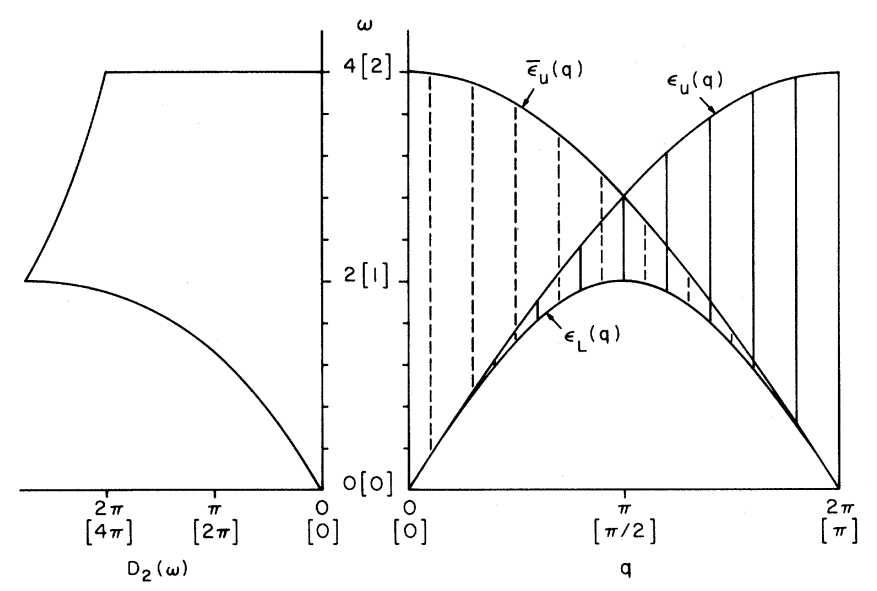

FIG. 5. Two-particle spectrum of TI model and $X Y$ model consisting of two partly overlapping continua with upper boundaries $\epsilon_{U}(q)$ and $\bar{\epsilon}_{U}(q)$, respectively, and the common lower boundary $\epsilon_{L}(q)$. Also shown is the density of two-particle states $D_{2}(\omega)$. Labels in square brackets are for the $X Y$ model, those without brackets are for the TI model. 
The two-particle excitations of both models (1.1) and (1.2) form a two-parameter continuum consisting of two partly overlapping sheets, as shown in Fig. 5. The energies of the two-particle excitations as a function of wave number $q$ and a parameter $k(0 \leq k \leq \pi)$ are

$$
\begin{aligned}
& {\left[\epsilon_{2}(k, q)\right]_{\mathrm{TI}}=2\left|\cos \left(\frac{k}{2}-\frac{q}{4}\right)\right|+2\left|\cos \left(\frac{k}{2}+\frac{q}{4}\right)\right|,} \\
& \left.\left[\epsilon_{2}(k, q)\right]_{X Y}=|\cos | k-\frac{q}{2}\right)|+| \cos \left(k+\frac{q}{2}\right) \mid .
\end{aligned}
$$

The two continua have upper boundaries given by

$$
\left[\epsilon_{U}(q)\right]_{\mathrm{TI}}=4\left|\sin \frac{q}{4}\right|,\left[\epsilon_{U}(q)\right]_{X Y}=2\left|\sin \frac{q}{2}\right|
$$

and

$$
\left[\bar{\epsilon}_{U}(q)\right]_{\mathrm{TI}}=4\left|\cos \frac{q}{4}\right|,\left[\bar{\epsilon}_{U}(q)\right]_{X Y}=2\left|\cos \frac{q}{2}\right|,
$$

respectively, and a common lower boundary given by

$$
\left[\epsilon_{L}(q)\right]_{\mathrm{TI}}=2\left|\sin \frac{q}{2}\right|,\left[\epsilon_{L}(q)\right]_{X Y}=|\sin q| .
$$

Thus, the functions $\Phi_{0}^{z z}(\omega)$ can be expressed in integrals over $q$ of functions $S_{\mathrm{zz}}(q, \omega)$, which are nonzero only within the boundaries of the two-particle spectra ${ }^{52}$;

$$
\begin{aligned}
{\left[S_{\mathrm{zz}}(q, \omega)\right]_{\mathrm{TI}}=} & \frac{\left[16 \cos ^{2}(q / 4)-\omega^{2}\right]^{1 / 2}}{8 \cos ^{2}(q / 4)} \theta(4|\cos (q / 4)|-\omega) \theta(\omega-2|\sin (q / 2)|) \\
& +\frac{\left[16 \sin ^{2}(q / 4)-\omega^{2}\right]^{1 / 2}}{8 \sin ^{2}(q / 4)} \theta(4|\sin (q / 4)|-\omega) \theta(\omega-2|\sin (q / 2)|)+4 \pi^{2} M_{z}^{2} \delta(q) \delta(\omega), \\
{\left[S_{\mathrm{zz}}(q, \omega)\right]_{X Y}=} & \frac{2}{\left[4 \sin ^{2}(q / 2)-\omega^{2}\right]^{1 / 2}} \theta(\omega-|\sin q|) \theta[2|\sin (q / 2)|-\omega] .
\end{aligned}
$$

The (normalized) density of two-particle excitations, defined by

$$
D_{2}(\omega) \equiv \int_{0}^{2 \pi} d q \int_{0}^{\pi} d k \delta\left(\omega-\epsilon_{2}(k, q)\right),
$$

can also be expressed in terms of elliptic integrals, ${ }^{41}$

$$
\begin{aligned}
{\left[D_{2}(\omega)\right]_{\mathrm{TI}}=} & 8 F\left\{\arcsin \left[\left(\frac{1}{2}\left\{1-\left[1-(\omega / 2)^{2}\right]^{1 / 2}\right\}\right)^{1 / 2} / k\right],\left[1-(\omega / 4)^{2}\right]^{1 / 2}\right\} \theta(\omega) \theta(2-\omega) \\
& +4 K\left\{\left[1-(\omega / 4)^{2}\right]^{1 / 2}\right\} \theta(\omega-2) \theta(4-\omega), \\
{\left[D_{2}(\omega)\right]_{X Y}=} & 2\left[D_{2}(2 \omega)\right]_{\mathrm{TI}} .
\end{aligned}
$$

This function, which is plotted in Fig. 5, has pronounced van Hove singularities at $\omega=0,2,4$ for the TI model and at $\omega=0,1,2$ for the $X Y$ model. This is precisely where the ACF's $\Phi_{0}^{\mu \mu}(\omega), \mu=x, y, z$, also have singularities. Hence, the singularities in $\left[\Phi_{0}^{z z}(\omega)\right]_{\mathrm{TI}}$ at $\omega=0,2,4$ given in (3.8) are the combined effect of the van Hove singularities in $\left[D_{2}(\omega)\right]_{\mathrm{TI}}$ and the singular behavior of the matrix elements $\left\langle G\left|S^{z}(q)\right| \lambda\right\rangle$ at the boundaries of the continuum of two-particle excitations. Here $|G\rangle$ is the ground state [see Eq. (1.9)].

In the $X Y$ case, the matrix elements $\left\langle G\left|S^{z}(q)\right| \lambda\right\rangle$ are constant, and therefore $\left[\Phi_{0}^{z z}(\omega)\right]_{X Y}$ is proportional to the two-particle density of states

$$
\Phi_{0}^{z z}(\omega)_{X Y}=(4 \pi)^{-1}\left[D_{2}(\omega)\right]_{X Y} \text {. }
$$

The singularities in $\left[\Phi_{0}^{x x}(\omega)\right]_{\mathrm{TI}}$ and $\left[\Phi_{0}^{y y}(\omega)\right]_{\mathrm{TI}}$ at $\omega=0,2,4$ and the singularities in $\left[\Phi_{0}^{\perp \perp}(\omega)\right]_{X Y}$ at $\omega=0,1,2$ are likely to be at least partly due to the two-particle excitations. By analogy, our results strongly suggest that the nonanalyticities at higher integer frequencies can be attributed to continua of $m$-particle excitations ${ }^{53}$ with energies,

$$
\epsilon_{m}\left(k_{1}, \ldots, k_{m}\right)=\sum_{l=1}^{m}\left|\epsilon_{1}\left(k_{l}\right)\right|, q=\sum_{l=1}^{m} k_{l},
$$

where the one-particle excitation energies $\epsilon_{1}\left(k_{l}\right)$ are given in (3.12).

An interesting property which holds for all of the $\Phi_{0}^{\mu \mu}(\omega), \mu=x, y, z$ in both the TI and the $X Y$ models at $T=0$ is that

$$
\frac{d^{2}}{d \omega^{2}} \Phi_{0}^{\mu \mu}(\omega)>0
$$

on the intervals between the singularities of these functions. Thus the ACF's are, $a$ fortiori, convex functions on these intervals of analyticity. A further consequence is that the ACF's have no smooth maxima, i.e., all maxima occur at points of nonanalyticity.

Finally, it is instructive to discuss available approximate results for $\left[\Phi_{0}^{x x}(\omega)\right]_{X Y}$ in view of the new exact results reported in this paper. The time-dependent ACF's obtained by calculations in the continuum approximation $^{39,43}$ (Luttinger model) have already been discussed in Sec. II. In frequency space, the Luttinger model yields the correct exponents for the leading and next-leading singularities of $\left[\Phi_{0}^{x x}(\omega)\right]_{X Y}$ at $\omega=0$, but it fails to reproduce any of the nonanalyticities at $\omega>0$. This demonstrates that the divergences and finite nonanalyticities of the ACF's at nonzero frequencies are intrinsic features of the 


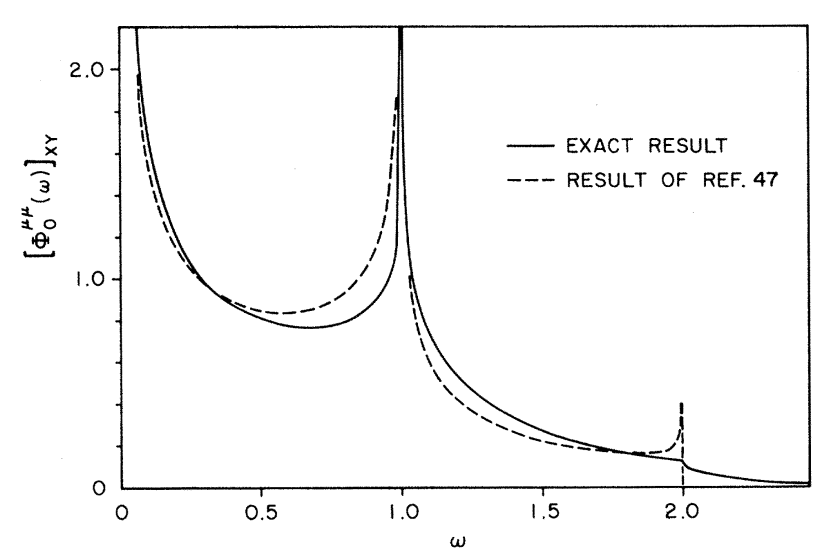

FIG. 6. Comparison of approximate result for frequencydependent ACF $\left[\Phi_{0}^{x x}(\omega)\right]_{X Y}$ from Ref. 47 (dashed line) with new exact result (solid line).

discrete quantum-spin model which cannot be accounted for by a continuum analysis.

In Ref. 47 an analytic expression for $\left[S_{x x}(q, \omega)\right]_{X Y}$ was conjectured on the basis of finite-chain calculations and sum-rule arguments. By construction, that approach neglects all spectral weight due to excitations lying outside the continuum of two-particle excitations. The ACF $\left[\Phi_{0}^{x x}(\omega)\right]_{X Y}$ obtained from the results of Ref. 47 is plotted in Fig. 6, together with the new exact result. We note that the overall qualitative agreement is fair for $\omega<2$. The approximate result yields the leading singularity with the amplitude correct within a relative error of $10^{-3}$. It correctly predicts another divergence at $\omega=1$, but an overly strong one, $\sim|\omega-1|^{-1 / 4}$, instead of logarithmic. At $\omega=2$ it predicts a further weak divergence followed by a cutoff instead of a square-root cusp.

In summary, we have analyzed the structure of the zero-temperature ACF's $\left\langle S_{0}^{\mu}(t) S_{n}^{\mu}\right\rangle, \mu=x, y$ for the 1D $S=\frac{1}{2}$, TI model at the critical field and the 1D $S=\frac{1}{2}$, isotropic $X Y$ model at zero field. We have combined these new analytic results governing the time dependence of the ACF's to calculate by Fourier transform the frequencydependent ACF's. We have found that the latter functions have singularities at an infinite set of frequencies, and we have determined the exact form of these singularities. Finally, the predictions of approximate calculations are reappraised in the light of the new exact results. Our new results bear strong relevance for low-temperature dynamical experiments on quasi-1D compounds such as the $X Y$-like substances $\mathrm{Cs}_{2} \mathrm{CoCl}_{4}$ and $\mathrm{PrCl}_{3}$ and the $S=\frac{1}{2}$ Ising-like substance $\mathrm{CsCoCl}_{3} \cdot 3 \mathrm{H}_{2} \mathrm{O}$.

\section{ACKNOWLEDGMENTS}

We would like to thank B. M. McCoy and J. H. H. Perk for many interesting discussions. One of us (G.M.) would also like to thank J. C. Bonner for useful comments. Some of the calculations performed herein were done using MACSYMA. This research was supported in part by the National Science Foundation under Grant No. PHY81-09910.

\section{APPENDIX}

Here we shall prove that the singularities in the frequency dependent ACF's $\Phi \Phi_{0}^{\xi \xi}(\omega)$ are determined by the long-time asymptotic behavior of the corresponding timedependent correlation functions $\Xi_{0}(t)$, and we shall present the detailed calculations of these singularities. We first recall that, by the definition of an asymptotic expansion of a bounded function $\left[\Xi_{0}(t)\right]_{\mathrm{LTAE}}$, for any $\delta>0$ there exists a $t_{\min }$ such that

$$
\left|\Xi_{0}(t)-\left[\Xi_{0}(t)\right]_{\text {LTAE }}\right|<\delta
$$

for $t>t_{\min }$. It is useful to define an auxiliary function,

$$
\begin{aligned}
\Psi_{\delta}^{\xi}\left(\omega ; t_{\min }\right)=\frac{1}{4} & \int_{-\infty}^{-t_{\min }} d t \Xi_{0}(t) e^{i \omega t} \\
& \left.+\int_{t_{\min }}^{\infty} d t \Xi_{0}(t) e^{i \omega t}\right] .
\end{aligned}
$$

As will be shown, the singularities of this function do not depend on $t_{\min }$. Furthermore, for values of $\omega$ where $\Psi \Psi^{\xi}\left(\omega ; t_{\min }\right)$ is finite, a controllably small error is made in approximating $\Xi_{0}(t)$ by $\left[\Xi_{0}(t)\right]_{\mathrm{LTAE}}$; that is, for any $\epsilon>0$ with

$$
\left|\Psi_{\delta}^{\xi \xi}\left(\omega, t_{\min }\right)-\left[\Psi \Psi^{\xi}\left(\omega, t_{\min }\right)\right]_{\mathrm{LTAE}}\right|<\epsilon,
$$

where $\left[\Psi \Psi_{0}^{\xi \xi}\left(\omega, t_{\min }\right)\right]_{\mathrm{LTAE}}$ is obtained from (A2) by replacing $\Xi_{0}(t)$ by $\left[\Xi_{0}(t)\right]_{\mathrm{LTAE}}$, there exists a $\delta$ and a $t_{\text {min }}$ such that

$$
\left|\Xi_{0}(t)-\left[\Xi_{0}(t)\right]_{\text {LTAE }}\right|<\delta
$$

for $t>t_{\min }$. Hence it is evident that the singularities of $\left[\Psi \Psi^{\xi}\left(\omega, t_{\min }\right)\right]_{\text {LTAE }}$, to be determined below, can be identified as the singularities of the frequency-dependent ACF

$$
\Phi \xi_{0}^{\xi}(\omega)=\Psi_{0}^{\xi \xi}\left(\omega, t_{\min }=0\right) \text {. }
$$

Let us then approximate $\Xi_{0}(t)$ by $\left[\Xi_{0}(t)\right]_{\mathrm{LTAE}}$, and accordingly delete the interval $\left(-t_{\min }, t_{\min }\right)$ from the Fourier transform (1.5). We consider first the calculation of $\Psi_{0}^{x x}\left(\omega, t_{\min }\right)^{(\mathrm{TI})}$. With the LTAE of (2.7), Eq. (A2) yields

$$
\begin{gathered}
{\left[\Psi_{0}^{x x}\left(\omega ; t_{\min }\right)\right]_{\mathrm{LTAE}}^{(\mathrm{TI})}=\frac{1}{4} \sum_{m=0}^{\infty} \sum_{n=0}^{\infty} A_{n}^{(x, m)} \lim _{u \rightarrow 0}\left[(i)^{m^{2}+2 n-1}(2 m-\omega)^{m^{2} / 2+n-3 / 4} \Gamma\left(\frac{3}{4}-\frac{1}{2} m^{2}-n,[i(2 m-\omega)+u] t_{\min }\right)\right.} \\
\left.+(i)^{1 / 2}(\omega-2 m)^{m^{2} / 2+n-3 / 4} \Gamma\left(\frac{3}{4}-\frac{1}{2} m^{2}-n,[i(\omega-2 m)+u] t_{\min }\right)\right],
\end{gathered}
$$

where $\Gamma(a, z)$ is the incomplete $\Gamma$ function, ${ }^{41}$ and

$$
A_{n}^{(x, m)}=\bar{A}(2 \pi)^{-m / 2} 2^{-m^{2} / 2-n} a_{n}^{(x, m)} .
$$

As $\omega \rightarrow 2 m, m=0,1,2, \ldots$, the function (A6) has singularities. Furthermore, the singular part of (A6) becomes in- 
dependent of $t_{\min }$. Therefore, the singularities of [ $\left.\Phi_{0}^{x x}(\omega)\right]_{\mathrm{TI}}$ depend only on the LTAE of $\left[X_{0}(t)\right]_{\mathrm{TI}}$, because $t_{\min }$ can be chosen arbitrarily large so that the LTAE is arbitrarily accurate. For the dominant singularity of (A6) we thus obtain the form of (3.2a). It is straightforward to prove that this property holds for $\left[\Phi \delta^{\xi}(\omega)\right]_{\mathrm{TI}}$ with $\xi=y$ and $z$ as well as $\xi=x$.

The same method can be used to calculate the singularities of $\left[\Phi_{0}^{\perp \perp}(\omega)\right]_{X Y}$ at $\omega=m, m=0,2,4, \ldots$ As before, they depend only on the LTAE of (2.9) of $\left[X_{0}(t)\right]_{X Y}=\left[Y_{0}(t)\right]_{X Y}$. The result for the dominant singularity at each frequency is given in (3.7a).

For the singularities of $\left[\Phi_{0}^{\perp \perp}(\omega)\right]_{X Y}$ at $\omega=m, m=1,3,5, \ldots$, the integral of (A2) with the LTAE of (2.9) leads to a different expression.

$$
\begin{aligned}
& {\left[\Psi_{0}^{\perp \perp}\left(\omega ; t_{\min }\right)\right]_{\mathrm{LTAE}}^{(X Y)}=\frac{1}{4} \sum_{m=0}^{\infty} \sum_{n=0}^{\infty} \frac{B_{n}^{(\perp, m)}(i)^{\beta_{n}(\perp, m)}}{\left[\beta_{n}^{(\perp, m)}\right] !}}
\end{aligned}
$$

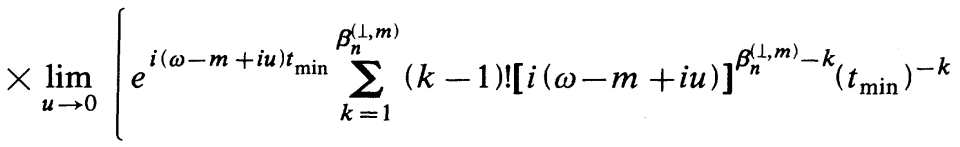

$$
\begin{aligned}
& -[i(\omega-m+i u)]^{\beta_{n}^{(1, m)}} \operatorname{Ei}\left[i(\omega-m+i u) t_{\min }\right]+e^{i(m-\omega+i u) t_{\min }} \\
& \times \sum_{k=1}^{\beta_{n}^{(\perp, m)}}(k-1) ![i(m-\omega+i u)]^{\beta_{n}^{(\perp, m)}-k}\left(t_{\min }\right)^{-k} \\
& \left.-[i(m-\omega+i u)]^{\beta_{n}^{(1, m)}} \operatorname{Ei}\left[i(m-\omega+i u) t_{\min }\right]\right),
\end{aligned}
$$

where $\operatorname{Ei}(z)$ is the exponential integral ${ }^{41}$ and

$$
\begin{aligned}
& B_{n}^{(\perp, m)}=(\bar{A})^{2} 2^{1 / 2}(2 \pi)^{-m / 2} b_{n}^{(\perp, m)}, \\
& \beta_{n}^{(\perp, m)}=\frac{1}{4}\left(m^{2}-1\right)+n .
\end{aligned}
$$

As $\omega \rightarrow m, m=1,3,5, \ldots$, the Ei function diverges log- arithmically. Except for the cast $m=1, n=0$, this logarithmic singularity is multiplied by power of $\omega-m$. All other terms in (A8) are nonsingular. Again, the singularities depend only on the LTAE (as do those of $\left[\Phi_{0}^{z z}(\omega)\right]_{X Y}$ ). The dominant singularity thus obtained for each frequency is given in (3.7b).
${ }^{1}$ T. Nakamura, J. Phys. Soc. Jpn. 7, 264 (1952); M. E. Fisher, Am. J. Phys. 32, 343 (1964).

${ }^{2}$ C. N. Yang and C. P. Yang, Phys. Rev. 150, 321 (1966); 151, 258 (1966).

3J. Des Cloizeaux and M. Gaudin, J. Math. Phys. ㄱ, 1384 (1966); M. Gaudin, Phys. Rev. Lett. 26, 1301 (1971).

${ }^{4}$ M. Takahashi and M. Suzuki, Prog. Theor. Phys. 48, 2187 (1972); M. Takahashi, ibid. 50, 1519 (1973); 1, 1348 (1974).

5J. D. Johnson and B. M. McCoy, Phys. Rev. A $\underline{6}$, 1613 (1972); J. D. Johnson, ibid. 9, 1743 (1974).

6J. D. Johnson and J. C. Bonner, Phys. Rev. B 22, 251 (1980).

${ }^{7}$ E. Lieb, T. Shultz, and D. Mattis, Ann. Phys. (N.Y.) 16, 407 (1961).

${ }^{8}$ S. Katsura, Phys. Rev. 127, 1508 (1962).

${ }^{9}$ M. Suzuki, Prog. Theor. Phys. 46, 1337 (1971).

${ }^{10}$ R. J. Baxter, Ann. Phys. (N.Y.) 70, 323 (1972).

11J. D. Johnson, S. Krinsky, and B. M. McCoy, Phys. Rev. A $\underline{8}$, 2526 (1973).

${ }^{12}$ D. C. Mattis, Theory of Magnetism I, Vol. 17 of Springer Series in Solid State Sciences (Springer, Berlin, 1981).

13J. H. Taylor and G. Müller, Phys. Rev. B $\underline{28}, 1529$ (1983).

${ }^{14}$ Time-dependent correlation functions of classical-spin systems are real functions at arbitrary temperature. This follows from Eq. (1.7b) rewritten as $\Xi_{n}^{*}(t)=\Xi_{n}(t-i \hbar \beta)$ in the classical limit $\hbar \rightarrow 0$.

${ }^{15}$ M. Steiner, J. Villain, and C. G. Windsor, Adv. Phys. $\underline{25}, 87$ (1976).

16J. P. Groen, H. W. Capel, J. H. H. Perk, T. O. Klaassen, and N. J. Poulis, Physica 97B, 126 (1979); J. P. Groen, T. O. Klaassen, N. J. Poulis, G. Müller, H. Thomas, and H. Beck, Phys. Rev. B 22, 5369 (1980).

${ }^{17}$ However, it does not hold for $\Phi_{n}^{\mu \mu}(\omega)$ with $n \neq 0$.

18B. M. McCoy, Phys. Rev. 173, 531 (1968).

${ }^{19}$ P. Pfeuty, Ann. Phys. (N.Y.) 57, 79 (1970).

${ }^{20}$ E. Barouch and B. M. McCoy, Phys. Rev. A $\underline{3}, 786$ (1971).

${ }^{21}$ T. Niemeijer, Physica $\underline{36}, 377$ (1967).

${ }^{22}$ S. Katsura, T. Horiguchi, and M. Suzuki, Physica 46, 67 (1976).

${ }^{23}$ T. N. Tommet and D. L. Huber, Phys. Rev. B $\underline{11}, 450$ (1975).

24J. H. H. Perk, H. W. Capel, and T. J. Siskens, Physica 89A, 304 (1977).

${ }^{25}$ M. W. Puga and H. Beck, J. Phys. C 15 , 2441 (1982).

${ }^{26}$ M. Mohan and G. Müller, Phys. Rev. B 27, 1776 (1983).

${ }^{27}$ B. M. McCoy, E. Barouch, and D. B. Abraham, Phys. Rev. A 
4, 2331 (1971).

${ }^{28}$ D. Ruelle, Statistical Mechanics (Benjamin, New York, 1969), p. 97; H. Araki, Commun. Math. Phys. 14, 120 (1969); H. W. Capel and J. H. H. Perk, Physica 87A, 211 (1977); J. H. H. Perk and H. W. Capel, ibid. 89A, 265 (1977).

${ }^{29}$ U. Brandt and K. Jacoby, Z. Phys. B 25, 181 (1976).

${ }^{30}$ A. Sur, D. Jasnow, and I. J. Lowe, Phys. Rev. B 12, 3845 (1975).

${ }^{31}$ W. Pesch and H. J. Mikeska, Z. Phys. B 30, 177 (1978).

${ }^{32}$ H. G. Vaidya and C. A. Tracy, Physica 92A, 1 (1978).

33J. H. H. Perk, Phys. Lett. 79A, 1 (1980).

${ }^{34}$ B. M. McCoy, J. H. H. Perk, and R. E. Shrock, Nucl. Phys. B 220, [FS8], 35 (1983); 220, [FS8], 269 (1983).

${ }^{35}$ G. Müller and R. E. Shrock, Phys. Rev. Lett. 1ㅣ, 219 (1983). Because of an error in composition at the journal, many of the entries in Table I of this reference were printed incorrectly. For example, $c_{4}^{(0)}$ should read $81 / 2^{7}$, not $(81 / 2)^{7} ; c_{1}^{(1)}$ should read $9 / 2^{3}$, not $(9 / 2)^{3}$; and so forth for all other entries of this type.

36J. Lajzerowicz and P. Pfeuty, Phys. Rev. B 11, 4560 (1975).

${ }^{37}$ In Ref. 35 the coefficients analogous to $a_{n}^{(x, m)}$ and $b_{n}^{(1, m)}$, called $c_{n}^{(m)}$ and $d_{n}^{(m)}$, respectively, were defined in such a way as to render $c_{0}^{(m)}=d_{0}^{(m)}=1$ by extracting an appropriate power of 2 . In the present work we have chosen a different notational convention. The connection between the two conventions is given by $a_{n}^{(x, m)}=2^{-\beta_{m}} c_{n}^{(m)}$ for the TI model and $b_{n}^{(1, m)}=2^{-\gamma_{m}} d_{n}^{(m)}$ for the $X Y$ model, where the right-hand sides of these relations refer to the notation of Ref. 35 .

${ }^{38}$ This is easily understood, since the leading term in the $m$ th tower of $\left[Y_{0}(t)\right]_{\mathrm{TI}}$ arises, for $m \neq 0$, from the operation of the derivative twice on the $e^{-2 i m t}$ factor in $\left[X_{0}(t)\right]_{\mathrm{TI}}$, with the accompanying $t^{-v}$ factor not being differentiated at all, so that the order in $t$ remains the same. However, for $m=0$, the derivatives must act on the $t^{-v}$ factor, and hence, they suppress the nonoscillatory tower in $Y_{0}(t)$ by two units relative to that in $\left[X_{0}(t)\right]_{\mathrm{TI}}$.

${ }^{39}$ A. Luther and I. Peschel, Phys. Rev. B $\underline{12}, 3908$ (1975).

${ }^{40}$ The special result $\left[Z_{0}(t)\right]_{\mathrm{TI}}$ was obtained by $\mathrm{T}$. N. Tommet and D. L. Huber, Phys. Rev. B 11,450 (1975), and the special result $\left[Z_{0}(t)\right]_{X Y}$ was given in Ref. 22. Note, however, that Eq. (27) of Ref. 22 is evidently incorrect for $g-m \neq 0$; see also L. L. Goncalves and H. B. Cruz, J. Magn. Magn. Mater. 15-18, 1067 (1980).

${ }^{41}$ I. S. Gradshteyn and I. M. Ryzhik, Tables of Integrals, Series, and Products (Academic, New York, 1965).

42J. Kurmann, G. Müller, H. Thomas, M. W. Puga, and H. Beck, J. Appl. Phys. 52, 1968 (1981).

${ }^{43}$ H. C. Fogedby, J. Phys. C 11,4767 (1978).

${ }^{44}$ D. C. Mattis and E. H. Lieb, J. Math. Phys. 6 , 304 (1965).

${ }^{45}$ This calculation of $\left[X_{0}(t)\right]_{\mathrm{TI}}$ was performed previously for Ref. 34.

${ }^{46}$ H. J. Mikeska, Phys. Rev. B $\underline{12}, 2794$ (1975).

${ }^{47}$ G. Müller, H. Thomas, M. W. Puga, and H. Beck, J. Phys. C 14, 3399 (1981); G. Müller, Phys. Rev. B 26, 1311 (1982).

${ }^{48}$ This is to be expected, since $S \rightarrow \infty$ is the classical limit, and $v \rightarrow-1$ in that limit; see, e.g., E. H. Lieb, Commun. Math. Phys. 31, 327 (1973); H. E. Stanley, in Phase Transitions and Critical Phenomena, edited by C. Domb and M. S. Green (Academic, New York, 1973), Vol. 3, p. 486.

${ }^{49}$ See, e.g., I. M. Gel'fand and G. E. Shilov, Generalized Functions (Academic, New York, 1968), Vol. 2.

${ }^{50}$ Parenthetically, a weaker constraint on the asymptotic behavior of $\Phi_{0}^{\mu \mu}(\omega)$ is imposed by sum rules (see Ref. 47), which require than any frequency moment of $\Phi_{0}^{\mu \mu}(\omega)$ be finite. This implies that, as $\omega \rightarrow \infty, \Phi^{\mu \mu}(\omega)$ falls faster than any power.

${ }^{51}$ Also parenthetically, for the $X Y$ case it has been noted that at any nonzero temperature $\left[\Phi_{0}^{z z}(\omega)\right]_{X Y}$ has a logarithmic divergence of the form $\sim e^{-J / k_{B} T}|\ln (\omega / J)|$ as $\omega \rightarrow 0$. This singularity is associated with a divergent density of states for transitions between thermally activated excitations. See, e.g., J. P. Groen et al., Phys. Rev. B $\underline{22}, 5369$ (1980).

${ }^{52}$ Note that the expressions for the same or related quantities found in Refs. 22 and 23 disagree with our results given in Eqs. (3.18) and (3.19).

${ }^{53}$ The role of $m$-particle excitations in the functions $\Phi_{n}^{x x}(\omega)$ and $\Phi_{n}^{y y}(\omega)$ of the general model described by Eq. (1.10) was previously discussed in Ref. 27. 